commutes, long work hours, and employer policies regarding health and safety. These non-traditional hazards have been associated with injury and illness, psychosocial stress, and unhealthy behaviors including poor diet and smoking. The cumulative impacts of both traditional and non-traditional hazards on the health and well-being of construction workers are largely unknown.

Methods We conducted annual surveys among apprentice construction workers to identify relationships between four study domains: work organization and environment, health behaviors, health outcomes, and work outcomes.

Results 963 baseline surveys were completed and returned by apprentice construction workers $(90 \%$ response rate, mean age 28). Preliminary analyses examined associations between work organization factors and four self-reported outcomes: lower work ability, lower productivity, higher rates of missed days of work due to injury, and use of prescription pain medication. We found that all four outcomes were associated with high job demands, low supervisor support, and low job security. Other factors associated with one or more outcomes included low job security, mandatory overtime, low coworker support, and low foreman supervision of safety. Compared to commercial construction workers, those in residential construction reported higher use of pain medication and higher rates of missed days due to work injuries. One year follow-up data from 901 workers are now being analyzed; we will present results of relationships between the four study domains.

Discussion Cross-sectional data highlight non-traditional worksite health risks, and suggest potential interventions to improve heath behaviors and outcomes among construction workers.

\section{E.3 EVALUATION OF THE NORWEGIAN AGREEMENT ON A MORE INCLUSIVE WORKING LIFE: SICKNESS ABSENCE IN INDIVIDUALS WITH MUSCULOSKELETAL AND PSYCHOLOGICAL DIAGNOSES}

Rachel L Hasting*, Therese N Hanvold, Suzanne L Merkus, Petter Kristensen, Ingrid S Mehlum. National Institute of Occupational Health, Oslo, Norway

\subsection{6/OEM-2019-EPI.29}

Objectives The Norwegian Agreement on a More Inclusive Working Life (the IW Agreement) was introduced in 2001 amid efforts to reduce sickness absence (SA) and increase work participation. Little research has been done on the effects of this agreement. The study's aim was to compare SA before and after introduction of the IW Agreement.

Methods Data from several national registries involving 115,119 individuals born in Norway 1967-1976 was used. Individuals were classified using ICPC-2 codes into musculoskeletal (code L) and mental health (code P) diagnosis groups. A difference-in-differences method using logistic regression was used to compare the difference in one year risk of SA lasting $>16$ days in individuals working in IW companies relative to non-IW companies in 2000 and 2005. Analyses were adjusted for age, gender, and industry. Standard errors were clustered at the individual level and average marginal effects were calculated with 95\% confidence intervals. Gender and industry specific models were also estimated.

Results There was no significant association between the IW Agreement and risk of SA in the overall musculoskeletal diagnosis group (-0.4 percentage points (PP), CI -0.9,0.1), but women had significantly lower risk of SA (-0.8 PP, CI -1.5,-
0.1). There was no association between the IW Agreement and risk of SA in the mental health diagnosis group (overall: $0.0 \mathrm{PP}, \mathrm{CI}-0.3,0.3)$. Significant associations were found in the electricity (males: 5.2 PP, CI 0.4,10.0) and wholesale/retail sectors (females: $-4.9 \mathrm{PP}, \mathrm{CI}-8.9,-1.0$ ) for musculoskeletal diagnoses, and in the financial/real estate sector (overall: 1.6 PP, CI 0.2,2.9, females: 3.1 PP, CI 0.2,5.9) for mental health diagnoses.

Conclusions Women in companies with an IW Agreement had a significant reduction in one year risk of SA for musculoskeletal diagnoses. Gender and industry may modify the association between the IW Agreement and SA in musculoskeletal and mental health diagnoses.

\section{$01 E .4$ ELECTRONIC WASTE RECYCLING IN QUÉBEC, CANADA: HIRING PRACTICES AND OCCUPATIONAL HEALTH AND SAFETY MANAGEMENT}

${ }^{1}$ Sylvie Gravel, ${ }^{2,3}$ Daniel Côté, ${ }^{4}$ Stéphanie Gladu, 2,5 France Labrèche*. ${ }^{1}$ School of Management Sciences, Université du Québec à Montréal (UQAM), Montréal, Canada; ${ }^{2}$ Institut de recherche Robert-Sauvé en santé et en sécurité du travail (IRSST), Montréal, Canada; ${ }^{3}$ Anthropology Department, Université de Montréal, Montréal, Canada; ${ }^{4}$ nstitute of Environmental Sciences, UQAM, Montréal, Canada; ${ }^{5}$ School of Public Health, Université de Montréal, Montréal, Canada

\subsection{6/OEM-2019-EPI.30}

Background and objective Electronic waste recycling (e-recycling) has received little attention from an occupational health and safety (OHS) perspective. Our objective was to describe hiring and OHS management practices in a sample of formal e-recycling facilities.

Methods Within a cross-sectional study of exposure of e-recycling workers to various contaminants, we conducted semistructured face-to-face interviews with a sample of 26 workers and 6 managers, employed in four companies. Thematic analyses, followed by a matrix analysis based on the companies' missions were conducted on the recorded interviews.

Results Three companies are small enterprises: one receives young offenders/ex-prisoners for up to six months of vocational internships; another is a private company recruiting its workforce through governmental programs integrating people with chronic health problems; the third, a family business, mainly employs workers within neighbouring communities. Lastly, a medium-sized unionized company recruits its employees through staffing agencies, offering permanent jobs to the best candidates after a three-month trial period. Most participants were male, aged between 20-50 years old, and had not completed high school, except for a few recent immigrants with graduate degrees. Regarding occupational hazards in their workplace, $40 \%$ of interviewees reported chemicals, $31 \%$ mentioned the danger of being struck by lift trucks, and less than $25 \%$ identified toxic vapours, inappropriate protective personal equipments (PPEs), cuts, dusts, musculoskeletal or back pain. Some workers expressed concern about the pace of work (and resulting stress), which they identified as an injury risk factor. None of the participants received any mentoring upon entering the job. Agency workers had inferior wages and did not have access to the same OHS preventive practices or PPEs as regular workers.

Conclusions In our sample, OHS management practices varied according to the employment relationship, although workers are exposed to similar working conditions. Working conditions in the growing e-recycling industry need our attention. 